\title{
MONTAGE INTERFÉRENTIEL POUR MESURER L'ÉPAISSEUR OPTIQUE DE LAMES MINGES TRANSPARENTES
}

\author{
Par Roger BERnARD et Jean-Glaude GaGon, \\ Laboratoire de Spectroscopie et de Luminescence de l'Université de Lyon.
}

\begin{abstract}
Résumé. - Un montage interférentiel à trois faisceaux, ne comportant aucune fente, permet de mesurer l'épaisseur optique de lames minces sans support, dans de bonnes conditions de luminosité. L'incertitude sur l'épaisseur réelle ne dépasse pas 50 angstroms. On obtient à volonté l'épaisseur en un point quelconque de la lame ou une valeur moyenne de l'épaisseur pour une zone circulaire d'une dizaine de millimètres carrés.
\end{abstract}

Abstract. - A three-beam interferometric device, without any slit, permits the measurement of the optical thickness of transparent unsupported films in conditions of good luminosity, with an accuracy of 50 angstroms. One obtains either the thickness at a given point of the film or an average value of thickness over a circular zone of about ten square millimeters.

Introduction. - Le problème que nous avons cherché à résoudre ici concerne le contrôle et la mesure de l'épaisseur de lames en formvar servant aux analyses par diffusion de protons [1]. Il ne s'agit donc pas de couches minces déposées sur un support, mais de lames libres, tendues seulement par leurs bords à la manière des lames de savon. Elles ont un indice de réfraction constant égal à 1,503 dans les conditions de nos expériences. Avec cette donnée, la mesure des épaisseurs se ramène donc à celle des trajets optiques. La méthode que nous avons choisie utilise les figures d'interférences entre trois faisceaux lumineux dont l'un traverse la lame à mesurer, les deux autres passant de chaque côté. Mais les dimensions de la lame, imposées par leur rôle ultérieur de cibles, nous ont forcés à écarter beaucoup les faisceaux latéraux, donc à produire des franges très fines qu'il a fallu observer avec un fort grossissement. Ainsi, nous avons dû modifier les montages classiques pour en augmenter la luminosité.

La méthode " des trois fentes " et son évolution. Considérons le montage de la figure 1. Les lentilles $\mathrm{L}_{1}$ et $\mathrm{L}_{2}$, de distances focales $f$, forment en $\mathrm{F}^{\prime}$ une image de la fente $\mathrm{F}$ qui émet des ondes lumineuses de longueur $\lambda$. Si l'on place entre $L_{1}$ et $L_{2}$ un écran opaque percé de trois fentes parallèles et équidistantes $F_{0}, F_{1}$ et $\mathrm{F}_{2}$, on obtient dans le plan $\mathrm{F}^{\prime} \mathrm{y}$ les franges d'interférences entre les trois faisceaux diffractés correspondants. En supposant ces fentes assez fines pour que la fonction de diffraction reste pratiquement constante dans le champ d'observation et en prenant pour unité l'intensité qui résulterait de l'éclairement de ce champ

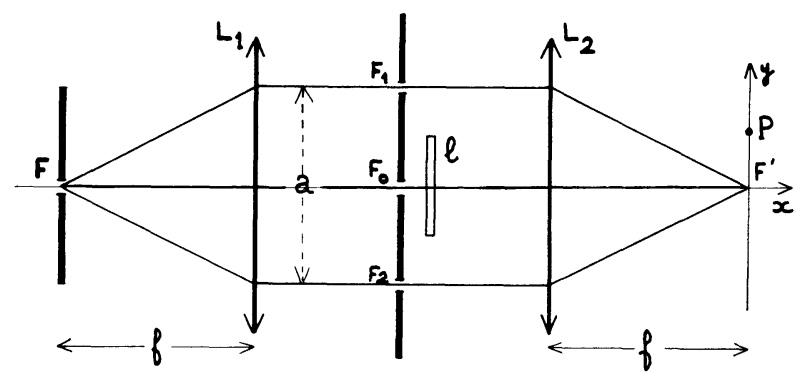

Frg. 1 - Montage à trois fentes pour la mesure des petites épaisseurs optiques (principe).

$\mathrm{F}$ : Fente source de lumière monochromatique.

$\mathrm{F}_{0}, \mathrm{~F}_{1}$ et $\mathrm{F}_{2}$ : Fentes diffractantes.

$\mathrm{L}_{1}$ et $\mathrm{L}_{2}:$ Lentilles convergentes.

$\boldsymbol{\ell}$ : Lame à mesurer.

par une seule des trois fentes, on peut exprimer l'intensité lumineuse le long de $\mathrm{F}^{\prime}$ y par :

$$
I=(2 \cos m y+1)^{2}
$$

où $m y$ représente le demi-déphasage entre les deux vibrations venues de $F_{1}$ et $F_{2}$ lorsqu'elles arrivent en un point $\mathrm{P}$, d'abscisse $y$ sur $\mathrm{F}^{\prime} \mathrm{y}$. La constante $m$ caractérise le montage et vaut $\pi a / f \lambda$. On observe donc dans le plan focal de $\mathrm{L}_{2}$ un système de franges d'intensités et de largeurs inégales (fig. 2).

Si maintenant on recouvre la fente centrale d'une lame transparente $\boldsymbol{l}$ qui allonge le trajet optique $\mathrm{F}_{\mathbf{0}} \mathrm{F}^{\prime}$ de $\delta=\lambda / 4$, la vibration venue de cette fente prend un retard de phase de $\pi / 2$ par rapport à la résultante 


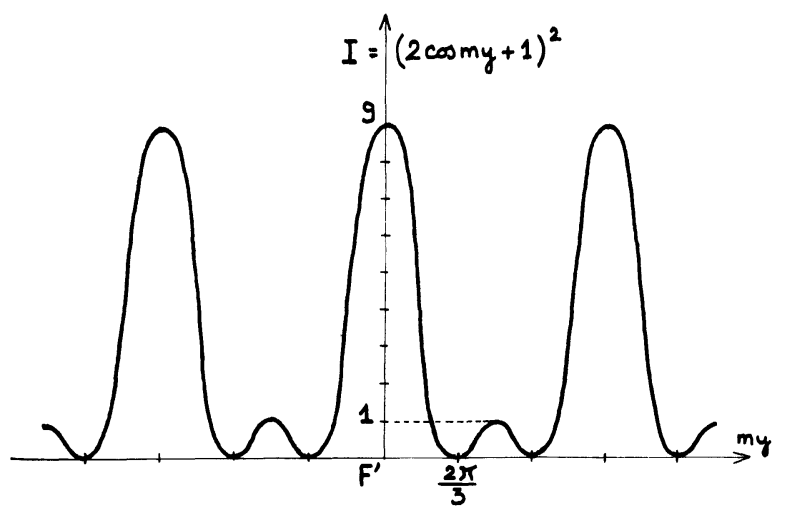

Frg. 2. - Répartition de l'intensité lumineuse le long de $F^{\prime} y$ pour trois vibrations d'amplitudes égales, en phase en $F^{\prime}$.

des deux autres. L'intensité lumineuse le long de $F^{\prime} y$ devient :

$$
I=(2 \cos m y)^{2}+1 .
$$

On observe alors des franges de Young superposées à un fond continu (fig. 3). Le même aspect apparaît

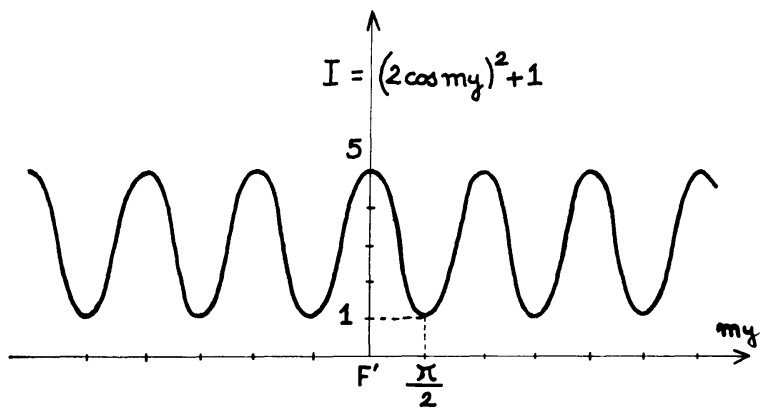

FIG. 3. - Nouvelle répartition de l'intensité lumireuse le long de $F^{\prime} y$, pour un déphasage de $\pi / 2$ de la vibration centrale par rapport aux deux autres. naturellement pour tous retards ou avances de phase multiples impairs de $\pi / 2$ (alors que pour les multiples de $2 \pi$ on retrouve l'aspect primitif de la figure 2 ).

Mais il faut remarquer que la lame $\boldsymbol{\ell}$, en retardant la vibration centrale par rapport aux deux autres, joue le même rôle qu'une lentille faiblement convergente placée sur l'ensemble des trois faisceaux. L'aspect primitif des franges qui a disparu du plan $\mathrm{F}^{\prime}$ y se trouve donc seulement déplacé en avant de ce plan. Entre les deux aspects que l'on vient de décrire, on observe, le long de l'axe $F^{\prime} x$, une évolution continue des franges dont le contraste s'inverse périodiquement. L'introduction d'une lame telle que $\boldsymbol{\ell}$ décale en bloc tous les systèmes de franges et la mesure du décalage permet de calculer l'épaisseur optique de la lame introduite, compte tenu des caractéristiques du montage utilisé.

F. Zernike [2] a contrôlé de cette façon l'épaisseur optique des lames qu'il préparait pour ses expériences sur le contraste de phase.

A. Lohmann [3], [4] a montré qu'on peut augmenter la luminosité du montage précédent en substituant aux trois fentes $F_{0}, F_{1}$ et $F_{2}$ les spectres d'ordre $0,+1$ et -1 d'un réseau de diffraction éclairé par un faisceau parallèle venu d'une fente source et d'un collimateur.

Inversement, A. Maréchal et ses collaborateurs [5] et P. Lostis [6] ont obtenu le même résultat en conservant les trois fentes diffractantes mais en supprimant la fente source.

En employant un laser pour produire la lumière cohérente, nous avons pu concilier les diverses améliorations apportées par ces auteurs à la méthode "des trois fentes » qui, ainsi, ne fait plus intervenir aucune fente.

Description et propriétés du montage. - Nous avons aligné sur un banc d'optique les éléments représentés sur la figure 4 .

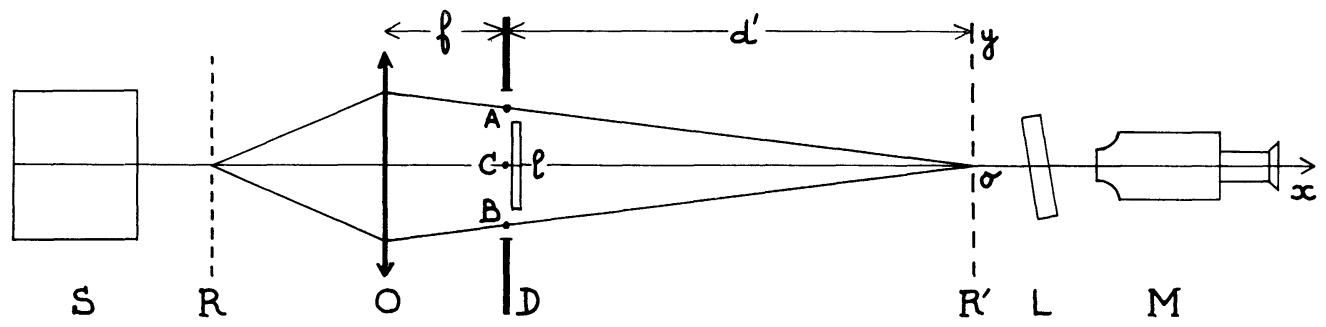

FIG. 4. - Montage réalisé (longueur totale : $4 \mathrm{~m}$ ).

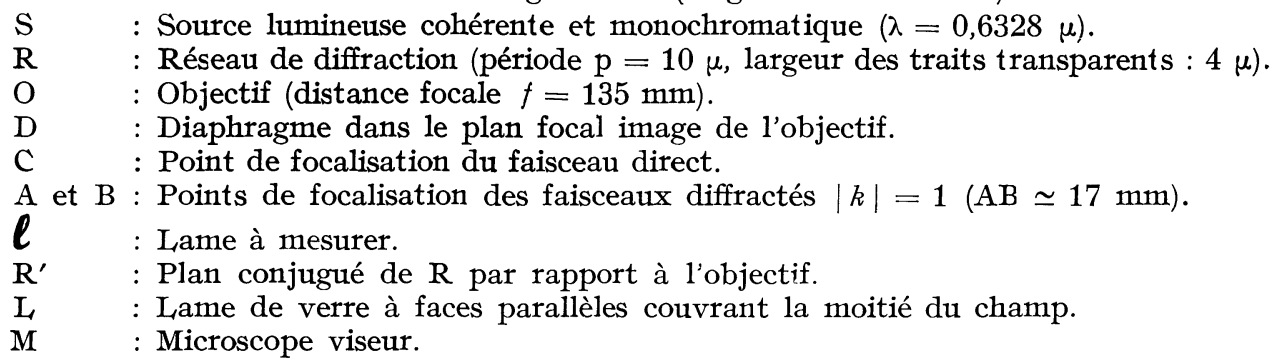


Un petit laser à gaz (hélium-néon) constitue une source commode de lumière cohérente, parallèle et monochromatique (S). Son faisceau tombe perpendiculairement sur un réseau en verre métallisé $\mathrm{R}$. L'objectif $O$ forme une image du réseau en $R^{\prime}$. Dans son plan focal, on a placé le diaphragme $D$ pour éliminer les faisceaux diffractés d'ordre $|k|>1$. L'image $\mathbf{R}^{\prime}$, ainsi privée de ses harmoniques supérieurs, se réduit à une figure d'interférences entre les trois faisceaux restants, d'ordre $0,+1$ et -1 . On observe les franges au moyen du microscope M. Dans le plan de visée, la lame à faces parallèles $L$ recouvre la moitié du champ d'observation. On incline cette lame de façon à amener les franges sombres de l'une des plages du champ dans le prolongement des franges brillantes de l'autre ( fig. 5), ce qui permettra par la suite un pointé précis de leur égalisation.

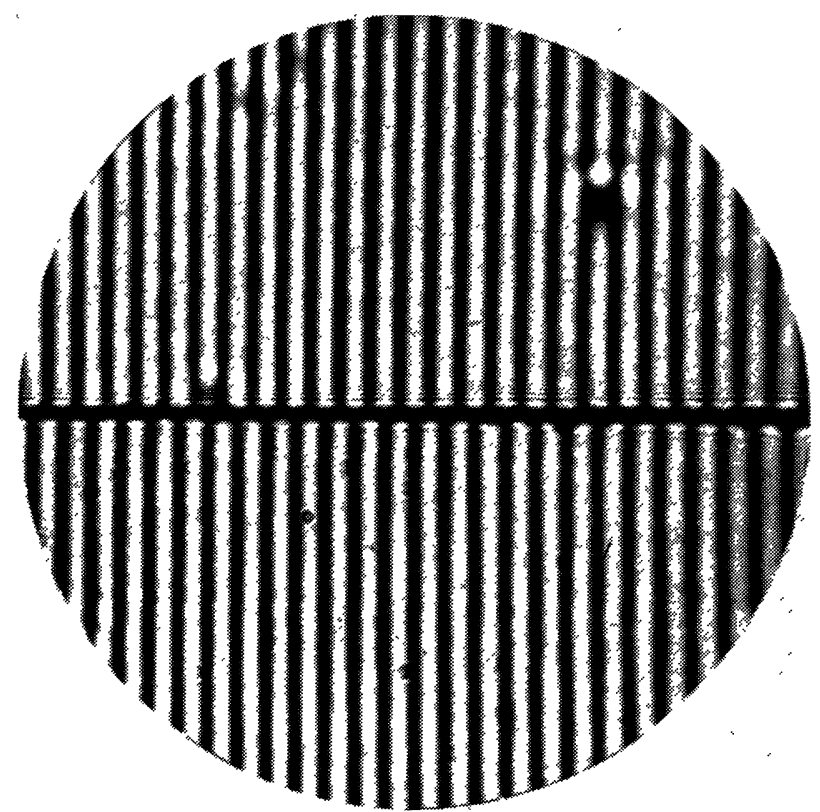

Fig. 5. - Champ d'observation partagé par le bord de la lame à faces parallèles en deux plages décalées d'un demi-interfrange.

Compte tenu des caractéristiques du réseau utilisé, on calcule que l'amplitude de la vibration centrale vaut sensiblement les $4 / 3$ de l'amplitude de chacune des deux autres. Dans la construction de Fresnel correspondante (fig. $6 \mathrm{a}$ ), on a pris arbitrairement l'unité la plus commode pour représenter les vecteurs amplitudes. Les deux vibrations diffractées arrivent en un point d'abscisse y dans le plan $\mathrm{Oy}$ avec un déphasage $2 m y$ où $m=2 \pi f \mid p \cdot d^{\prime}$. L'intensité lumineuse le long de Oy vaut donc ( fig. 7 a) :

$$
I=(3 \cos m y+2)^{2} \text {. }
$$

Si maintenant on donne un déphasage-retard de $\varphi$ à la vibration centrale par rapport aux deux autres

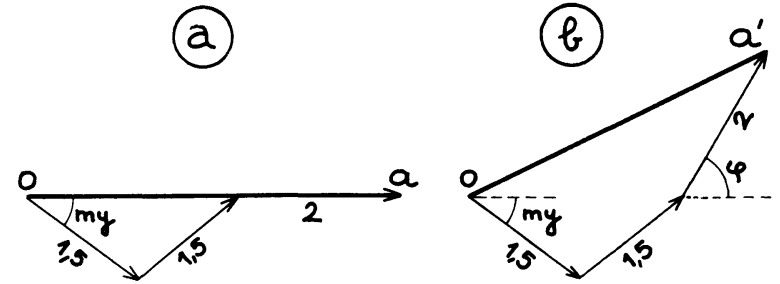

FIG. 6. - Construction de Fresnel donnant 1'amplitude résultante le long de $\mathrm{Oy}$ :

a) dans le plan $R^{\prime}$ en l'absence de lame $\ell(\mathrm{Oa})$,

b) dans un autre plan que $R^{\prime}\left(\mathrm{Oa}^{\prime}\right)$, ou dans le plan $R^{\prime}$ en présence de lame déphasante.

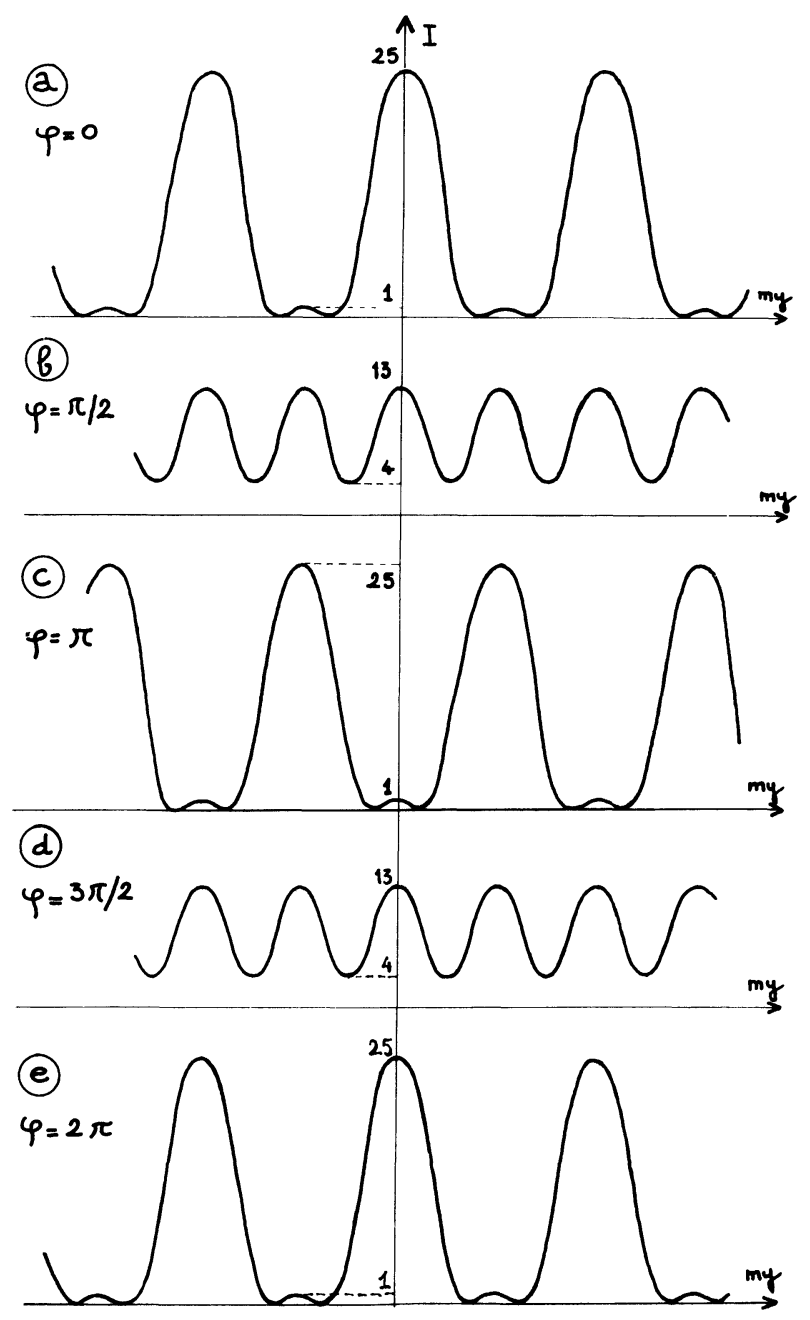

FIG. 7. - Répartition de l'intensité lumineuse le long de Oy pour quelques valeurs de $\varphi$ :
a) $\varphi=0 \quad ; \quad I=(3 \cos m y+2)^{2}$
b) $\varphi=\pi / 2 ; \quad I=9 \cos ^{2} m y+4$.
c) $\varphi=\pi \quad ; \quad I=(3 \cos m y-2)^{2}$.
d) $\varphi=3 \pi / 2 ; \quad I=9 \cos ^{2} m y+4$.
e) $\varphi=2 \pi ; \quad I=(3 \cos m y+2)^{2}$. 
en introduisant la lame à mesurer sur le faisceau direct, ou bien en visant un plan situé en arrière de $\mathrm{R}^{\prime}$, l'intensité devient, d'après la construction de la figure $6 \mathrm{~b}$ :

$$
I=(3 \cos m y+2 \cos \varphi)^{2}+4 \sin ^{2} \varphi .
$$

On a représenté ses variations pour quelques valeurs particulières de $\varphi$ sur les figures $7 \mathrm{~b}$ à $7 \mathrm{e}$. On retrouve donc ici les variations périodiques de l'aspect des franges qui caractérisent les déphasages introduits sur le faisceau central.

Mesure des lames. - Comme dans la méthode de Zernike, le premier temps de la mesure consiste à viser un système de franges bien déterminé au moyen du microscope. On obtient la meilleure précision sur ce pointé avec un aspect du type $7 \mathrm{~b}$ au voisinage duquel on décèle facilement l'inégalité d'intensité des franges [7].

Puis on place la lame à mesurer, d'indice $n$ et d'épaisseur $e$, sur le faisceau central et perpendiculairement à celui-ci. Le système de franges visé passe de $\mathrm{P}$ en $\mathrm{P}^{\prime}($ fig. 8) et il faut avancer le microscope le

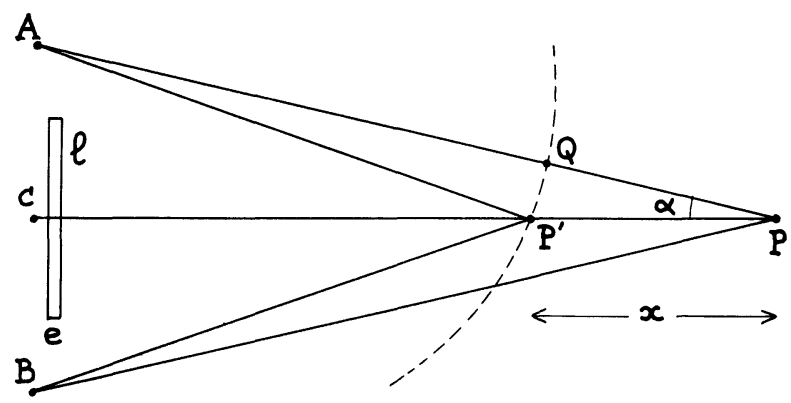

FIG. 8. - Décalage $\mathrm{PP}^{\prime}$ du plan de visée compensant le déphasage produit par la lame $\boldsymbol{\ell}$.

(Pour la clarté de la figure, on a dessiné l'angle $\alpha$ beaucoup plus grand que dans la réalité.)

long du banc d'optique pour retrouver l'aspect précédemment pointé. Établissons une relation entre le déplacement $x$ du microscope et l'épaisseur cherchée.
Comme le déphasage entre les vibrations venues de A et de $\mathrm{C}$ doit rester le même, on a :

$$
\mathrm{AP}-\mathrm{CP}=\mathrm{AP}^{\prime}-\left[\mathrm{CP}^{\prime}+e(n-1)\right] .
$$

Or :

et :

$$
\mathrm{AP}^{\prime}=\mathrm{AQ} \# \mathrm{AP}-x \cdot \cos \alpha
$$

d'où :

$$
\mathrm{CP}^{\prime}=\mathrm{CP}-x
$$

L'angle $\alpha$ vaut environ $10^{\prime}$ dans notre montage. On peut donc réduire $\cos \alpha$ aux deux premiers termes de son développement. L'équation précédente fournit alors la relation cherchée : $e=\alpha^{2} \cdot x / 2(n-1)$.

On peut connaître $\alpha$ à quelques millièmes près en mesurant l'interfrange $i$ en $\mathrm{P}$ après obturation du faisceau central. On obtient alors un système de franges de Young pour lequel $i=\lambda / 2 \alpha$. Par ailleurs, on connaît l'indice $n$ à deux millièmes près. La principale incertitude concerne la mesure de $x$. Elle atteint $0,4 \mathrm{~mm}$ en valeur absolue, alors que $x$ vaut environ $9 \mathrm{~mm}$ pour une lame de 1000 angstroms d'épaisseur. On fait donc une mesure à $5 \%$ près dans ce cas. La précision augmente naturellement pour des lames plus épaisses, mais il faut remarquer que la méthode ne s'applique directement qu'aux lames assez minces pour produire un déphasage inférieur à $\pi$. Pour les autres, il y a lieu de déterminer d'abord approximativement l'épaisseur optique pour savoir combien de demi-longueurs d'onde il convient d'ajouter au résultat.

Notons enfin que le montage décrit ici se prête à deux usages distincts :

1) En plaçant la lame à mesurer dans le plan focal de l'objectif, on obtient son épaisseur en un point. La planéité de la lame doit alors se traduire par un aspect constant du système de franges lorsqu'on déplace la lame dans son propre plan.

2) La lame placée un peu plus loin sur l'axe optique reçoit de la lumière sur $10 \mathrm{~mm}^{2}$ environ de sa surface. On obtient dans ce cas l'épaisseur moyenne d'une lame pour laquelle on n'a pas besoin d'une excellente planéité.

Manuscrit reçu le 18 mars 1967.

\section{BIBLIOGRAPHIE}

[1] Luthereau (F.) et Tousseit (J.), C. R. Acad. Sci., 1967, 264, 77-80.

[2] Zernike (F.), J. Opt. Soc. Amer., 1950, 40, 326-328.

[3] Lohmann (A.), Z. Physik, 1956, 143, 533-537.

[4] Lohmann (A.), Optica Acta, 1962, 9, 1-12.
[5] Maréchal (A.), Dupuy (O.) et Renault (M.), Optica Acta, 1962, 9, 47-54.

[6] Lostis (P.), J. Physique, 1964, 25, 118-124.

[7] Reinet (C.) et Viénot (J.), Rev. Opt., 1953, 32, 27-33. 